\title{
Air Light Estimation Algorithm by using Fuzzy based Dark Channel Prior
}

\author{
Simranjit Kaur \\ M.Tech Student, SSIET \\ Patti, Punjab, India-143416
}

\author{
S. A. Khan, PhD \\ Associate Professor \\ SSIET, Patti, Punjab
}

\author{
Rajwant Kaur \\ M.Tech Students \\ SSIET, Patti, Punjab
}

\begin{abstract}
The unwanted environmental circumstances decrease the visibility and hidden information of theremotely sensed images. Since visibility is a significant quality issue in these images, thus, visibilityimprovement methods are necessary for improving the significant details of remotely sensed images. Thispaper has proposed a novel technique for improving the visibility of outdoor images. Theproposed method produces efficient results by using fuzzy filter based dark channel prior. The fuzzy filter can automatically extract the local atmospheric light and roughly eliminate theatmospheric veil in local detail enhancement. The proposed technique is designed and implemented usingin MATLAB with the help of image processing toolbox. The qualitative results have clearly show that theproposed image enhancement technique can preserve significant detail of the original image.
\end{abstract}

\section{Keywords}

Dark channel prior, Haze, Airlight,Fuzzy filter.

\section{INTRODUCTION}

Owing to consumption and hanging environmental contaminants in a haze, outdoor pictures get weak visibility within rainy and windy weather. Poor visibility affects not only client pictures but also computer vision system used for outdoor situations, like object detection in addition to online video monitoring [1]. Haze elimination, which can be known as dehazing, is known as a critical approach due to the fact haze-free graphics usually look attractive and may even noticeably boost the whole process of computer visual performance. Strategies provided in the course of earlier reports attained several graphics to perform dehazing. For instance, polarization-based techniques utilize the polarization property associated with dispersed light to revive the scene level info through at least two graphics obtained with various levels of polarization [14]. In the same manner, several graphics of the same scene are captured in different weather conditions so that you can utilize as reference graphics which are clicked in perfect weather.

\subsection{Fuzzy Filter}

In Fuzzy filter, new outcomes are generated with the help of actual addition of measured real vectors (i.e., differential variations), and computed by calculating the difference through partners connected with others obtained from the population. [7]. The new solution (i.e., the children) are preserved in the subsequent generation after they outperform the related parents (i.e., should they be according to the improved fitness), particularly, this processing pattern is performed around Fuzzy filter. The three operators are implemented within the supplied information: first restoration, after which cross-over, last but not least selection [8]. The main difference around Differential trend regarding genetic algorithms is frequently restoration. For each of current population, $\mathrm{r}=1,2, \ldots \mathrm{N}$, where $\mathrm{r}$ is the iteration index, a new mutant vector $v_{r}^{l}$, will be created as follows:

$$
v_{r}^{l}=x_{r 1}+F *\left(x_{r 2}-x_{r 3}\right)
$$

Where $r_{1}, r_{2}, r_{3}$, are chosen randomly, and all are distinct. $\mathrm{F} \in[0.2]$ is the scaling factor. $x_{r 1}, x_{r 3}$ are donor vectors.

\subsection{Haze}

Haze removal is definitely an important concept in the image processing domain. Pictures of outside scenes are normally disturbed by the atmospheric moisture, dust, smoke, water droplet etc. These all are reason to create air pollution that usually called Haze. All atmospheric particles have ranging below of $1000 \mathrm{~m}$ and haze intensity is 4 to 10 . When atmospheric particles suspended around the sky haze produced. The formation of the hazy image is mainly influenced by the fact that the light from the camera source is scattered by water droplets in the sky.Scattering is occurred by two statically phenomenon namely attenuation and air-light

Haze $=$ Attenuation + Air-light, the equation can be Expressed as

$$
I(x)=j(x) \cdot t(x)+A(1-t(x))
$$

\subsection{Bilateral filtering}

Filtering boosts photos without the need of affecting edges, by using your non-linear mix of close by picture values. This technique makes use of bilateral filter that is a non-linear edgepreserving and also disturbance decreasing smoothing filter. This technique needs pre and also posting running steps. With the pre running Histogram equalization is done. This pre running raises the distinction in the picture prior to a errors treatment and results in the more effective opinion involving oxygen light map. Histogram stretching out can be used since an article running, which often grows distinction in the errors no cost image. Transfer purpose of the particular stretching out is attuned in accordance with the picture content [2].

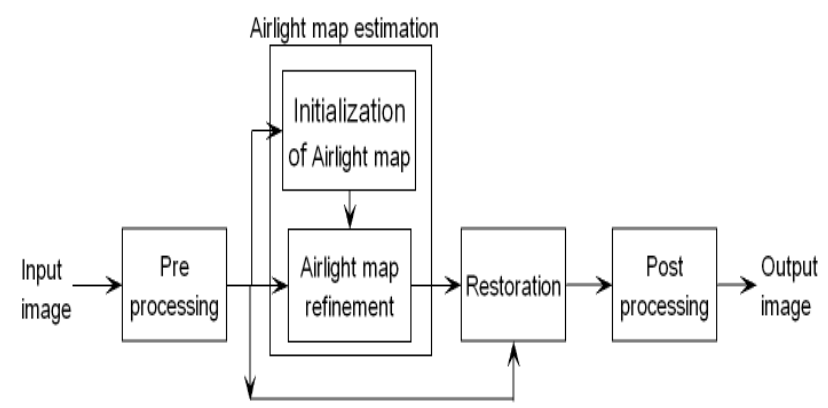

Fig 1: Block diagram showing bilateral filtering for removal algorithm

After the pre processing initial value of air light map is estimated. Air light map A is a scalar quantity and is always positive. This is represented as:Where is the image intensity in the absence of fog is the global atmospheric constantFor fog free image, except for sky region intensity of the dark channel is low and tends to be zero. So and positive, then 


\subsection{Dark channel prior}

Dark channel prior is a study of outside photograph haze removal. It can be in line with the indisputable fact that any local sections from the haze free of charge photos as their intensity can be surprisingly low in a minimum of one colour channel. This information is used by dim funnel before approach to increase the quality of photograph [5]. From these $p$ as their intensity can be reduced this breadth of your haze is often calculated in addition to our prime quality haze free of charge photograph is often recovered.The atmospheric light A was estimated from foggy image by using dark channel prior with a fixed patch size. Firstly, for selecting the patch size rectangle is used. Secondly, local dark channel is computed to estimate the atmospheric light in the rectangle.

\subsection{Trilateral Filtering}

This specific filtration smooth's illustrations or photos without having impacting perimeters, by way of the non-linear mix of neighbourhood photo values. Around the heap filter switches every pixel by weighted averages of that neighbour's pixel. The load allotted to each neighbour pixel lessens having the range in the photo airline and also the range to the severeness axis. This filter allows us to obtain result quicker while rival other.

\subsection{Fuzzy Filtering}

The idea driving this filtering is usually to normal your pixel using additional pixel ideals by reviewing the neighbourhood, however simultaneously to address critical image structures just like edges. The main priority from the recommended filtering is usually to distinguish between regional variations as a result of noises in addition to as a result of image structure. Your order to accomplish this, each pixel all of us derive a price in which communicates their education where the mixture within a particular path is usually small.

\subsection{Contrast Limited Adaptive Histogram Equalization}

Difference constrained flexible histogram equalization is frequently abbreviated while CLAHE. Inside this method absolutely no previous weather data is needed to progress a quality of the image. Within this a grabbed photo can be converted through RGB (Red, Inexperienced plus Blue) colouring space or room on the HSI (Hue, Saturation plus intensity). [6] This specific conversion process is performed since HSI colouring space or room senses a colors just like the human eye. A HIS shade style defines just about every shade together with some factors:

\section{LITERATURE REVIEW}

Wei, et al. (2013)provided theParticle Swarm Optimization techniquebased application to solve image processing problem. The enhancement method is a non-linear optimization difficulty with many constraints. The main aim of the proposed PSO need to increase an fuzzification value in order to deliver better the contrast and detail of image.Tripathiet al.(2012)[2] introduced pattern recognition having objective to classify objects into various classifications. They work on effective optimization method called Particle Swarm Optimization in the field of pattern recognise and image processing. In this proposed process the credit of PSO parameters is evaluated based on the interpretation of suggested algorithms.Chen, et al. (2009) [12] suggested a PSO based hue preserving colour picture improvement method. The intensity transformation process use local and global information details of the input picture and the fuzzification take the entropy and edge details to calculate the picture clarity. So, the proposed method gives better results.Xu Haoran, et al. (2012) [4] explained that Outside view and pictures reduce the quality under poor weather conditions, and produce Hazy images. The scientific study is done on various proposed techniques to improve the visibility of hazy image, which mainly worked on saturation and brightness. This paper used polarization and RETINEX based approach which makes dehazing simpler.Xu, Zhiyuan, et al. (2010 [10]Surveyed various haze removal techniques are studied and compared. Many efficient haze removal algorithms are so far proposed.. The main task here is to overcome the disadvantages of the earlier techniques of picture processing application.Desaiet al.(2009[14]presented an improved restoration model based dark channel prior. The proposed technique has redefined transmission map, with the aim to reduce the color distortion problem. The modified joint trilateral filter is also utilized to improve the coarse estimated atmospheric veil. It provides visually significant haze-free images and also preserves the significant detail. Yu, Jinget al. (2010) [8] presented a modified gain coefficient filter based Dark channel prior (DCP) for single image dehazing. To reduce the color distortion, restoration model of DCP is also redefined. Chu, Chao et al. (2010) [7] proposed ideal prior known as dark channel prior (DCP) that has brought a fantastic attention. Based on the DCP, the specific dehazing is going to be gained as a result of some essential strategies: atmospheric light estimation, transmission map estimation, transmission map refinement, and image reconstruction. This particular dehazing method gives a step-bystep technique of complicated solutions inside ill-posed inverse problem. It will eventually facilitate advancement of innovative dehazing algorithms. Guo et al.(2010)[6] presented a unique fog removal factor selection algorithm centered on genetic algorithm (GA). It concentrated on the approach to choose optimum constants for image defogging. This method put on two main fog removal algorithms by selecting main constants and then optimizes them using the genetic algorithm. Wolfeet al. (10) [11] offered imaging filter along with darker channel prior to fix the problem together with halo effects. However, all this filtration generate significant computational pressure however the dehazing consequence in the dark channel prior suitable for improvement.

\section{GAPS IN LITERATURE SURVEY}

Fog removal algorithms become more beneficial for numerous vision applications. It has been observed that the most of the existing research have mistreated numerous subjects. Following are the various research gaps concluded using the literature survey:

Subsequent section contains various gaps found in existing literature are:-

1. The primary drawback of majority of existing methods is that it may lose significant detail of restored images with large haze gradients.

2. The majority of existing haze removal techniques has taken restoration value statically, that depends upon the given set of images. Which limits the performance of haze removal as restoration value needs to be adaptive as effect of haze on given image varies scene to scene and atmospheric veil.

3. In existing literature not much work is done to improve the coarse estimated atmospheric veil by using improved/ hybrid variants of filters. 


\section{PROPOSED HAZE REMOVAL APPORACH}

We develop a method which combines the two existing methods of fog removal and bilateral filter is applied to it. It is found that the proposed method is more suitable for obtaining the better quality of the image than the most of the existing methods. The results produced by the existing dark channel prior method have less PSNR value and more Mean square error. Therefore the overall objective is to improve the results by combining CLAHE with Dark channel prior method. The proposed algorithm is designed and implemented in MATLAB using image processing toolbox.The detailed algorithm for the proposed approach is given below:

There are various phases through which we can get the clear fog free image. These are as following:-

Phase1: To start the whole process, we have to take a foggy input image on which differential algorithm and other techniques will be applied to get the high-quality image [7].

Phase2: Generate random population for Fuzzy filter. Here, Fuzzy filter is used to optimize the image parameters on which the clarity of image mostly depends.

Phase3: Fuzzy filter works according to the fuzzification. In proposed work, there is fuzzification function in which two parameters are used to select an optimal generation.

$\mathrm{MOF}=w_{1} * \mathrm{CG}+w_{2} *\left(\frac{1}{P S P}\right)$

Where CG is contrast gain, PSP is the percentage of saturated pixels and $w_{1}, w_{2}$ are weighted factors. This function selects those values where contrast gain is high and saturated pixels are less.

Phase4: Repeat the steps 5 to 7 until stopping criteria not met.

Phase5: Apply the restoration operator which adds difference vector(s) to a base individual to explore the search space as follows:

$$
D_{i, G+1}=x_{c 1, G}+F\left(x_{c 2, G}-x_{c 3, G}\right)
$$

where $c_{r 1}, c_{r 2}, c_{r 3}$ are distinct. $\mathrm{F}$ is the restoration factor and $D_{i, G+1}$ is the donor vector which is responsible for further generation.

Phase6:Apply recombination operator which often merge productive solutions coming from the earlier generation with current donors [7].

$T_{j, i, G+1}=\left\{D_{j, i, G+1}\right.$ ifrand $_{j, i} \leq$ CRor $\left.j=i_{\text {rand }}\right\}$

else

$T_{j, i, G+1}=x_{j, i, G}$

where $T_{j, i, G+1}$ is trial vector, CR is cross over ratio and $x_{j, i, G}$ is target vector.

Phase7:Apply selection operator to select the optimum parameter. The greedy scheme is crucial for fast convergence of DE.

$x_{i, G+1}=\left\{T_{i, G+1}\right.$ if $T_{i, G+1} \leq f\left(x_{i, j}\right\}$

Otherwise

$x_{i, G+1}=x_{i, G}$ here, $x_{i, G+1}$ is the best individual.

Phase8:Once the stopping condition met means when there is optimal population is available, then apply dark channel prior. Dark channel means when the intensity of pixels in one or more color channels is low or close to zero [13].

$I_{d a r k}(x)=\min _{c \in\{r, g, b\}}\left(\min _{y \in \Omega(\mathrm{x})}\left(C_{c}(y)\right)\right)$

where $C_{c}$ depicts, a color channel of I and local patch placed on $\mathrm{x}$ is defined by $\Omega(\mathrm{x})$.

Phase9: Evaluate transmission of the source picture using dark channel prior. After DCP, transmission map is estimated $\mathrm{t}(\mathrm{x})$ intended for continuing further with the solution. Now to normalize (2) divide both sides by A

$\frac{I^{c}}{A^{c}}(x)=\mathrm{t}(\mathrm{x}) \frac{J^{c}}{A^{c}}(x)+1-\mathrm{t}(\mathrm{x})$

Phase10: Evaluate airlight map of the input image using the transmission map image.

$A=\max (\min (t, 1), 0)$

Phase11: Now apply gradient based recursive bilateral image filter.

A recursive bilateral filter is a non-linear, edge-preserving as well as disturbance decreasing and also removing technique for pictures. An intensity value of each pixel in a perception can be relocated by way of calculated weighted average value from other neighborhood pixels [9]. This preserves the edges using systematically looping using each pixel as well as changing weights for the surrounding pixels accordingly. The bilateral filter is defined as

$A(x, y)=\min _{c \epsilon(r, g, b)}\left(I^{c}(x, y)\right)-\min _{c \in(r, g, b)}\left[I^{c}(x, y)\left(1-\frac{A(x, y)}{I_{\infty}}\right)\right]$

Where

$I^{\boldsymbol{c}}$ depicts the picture intensity in clear weather

$\boldsymbol{I}_{\infty}$ is a global atmospheric constant

Phase12: Evaluate refined transmission map so called coarse estimated atmospheric veil [1].

a. For the initial picture minimum value of the extended dark channel is calculated.

$d_{c e}(i+m, j+m)=\min (I(i, j,:))(15)$

where $d_{c e}$ is the extended dark channel and $m$ represents the mask

b. Then for the transmission map t the array containing all ones is created. Now calculate the input depth by using following equation

$t=1-W * \frac{d c o}{A}$

Here, $\mathrm{t}$ is transmission map, and Dco is an original dark channel.

$t=\max (\min (t, 1), 0)$

c. Then , the input depth of dark channel for the original is measured by using the given equation:

$d_{c o} 1=\min (\min (\min (I(:,:, ;))))$; 
d. Now input depth is filtered with the help of given equation:

$t 1(i, j)=\left(d c_{\max }-d c_{\text {ori }}\right) *(A-\min (I(i, j,:)))$

$t 2(i, j)=\left(d c_{\max }-d c_{\text {ori }}\right) * A-\left(\min (I(i, j,:))-d c_{\text {ori }}\right) *$

$\min (I(i, j,:))$

$t(i, j)=\frac{t 1(i, j)}{t 2(i, j)}$

where $d c_{\max }$ is maximum dark channel, $d c_{\text {ori }}$ is original dark channel and A represents atmospheric light.

Phase13: Now apply restoration model to return the haze free image [13]. A Clear image can be restored as follows:

$o p(j, l, i)=\frac{I(j, l, i)-A}{\max (t(j, 1), t 0)}+A$

here $t_{0}$ is lower bound which reduce the color distortion, and halo artifacts and op is a haze free image.

Phase14: Evaluate performance metrics for haze free image.

\section{EXPERIMENTAL RESULTS}

Figure above has shown the input images for experimental analysis. In this section we will compare the results of the images by the existing and the proposed approaches. The images of the existing and the proposed approaches are shown as under
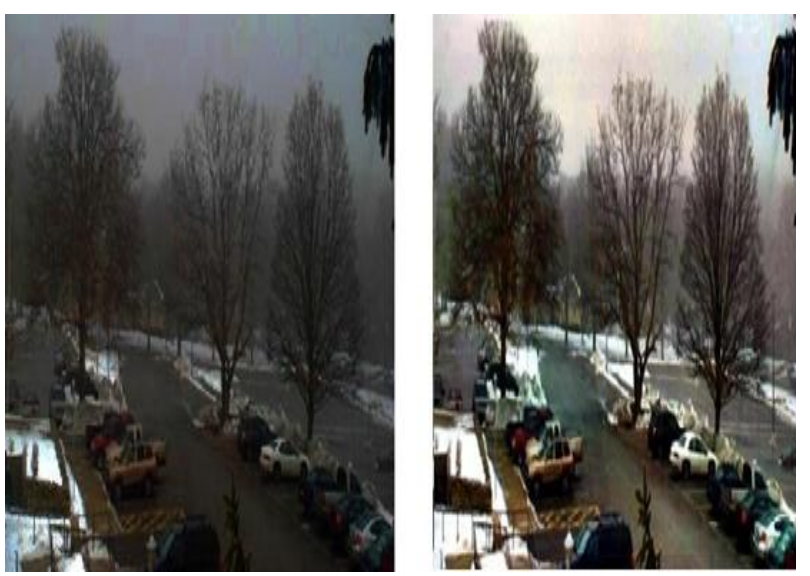

Figure 5.1(a) Restored Image by Existing Method (b) Restored image by proposed Method

Fig.5.1 (a) is showing the restored image by the existing approach and fig.5.1 (b) is showing the restored image by the proposed method. The figure 5.1(b) gives the better results as compared to the figure5.1 (a).

\section{PERFORMANCE ANALYSIS}

To demonstrate the effectiveness of the proposed technique, five foggy images are taken. The proposed technique and existing technique of Fan Guo are considered forcomparative analysis.

MSE (Mean Squared Error) -Mean square error will be to compute one indication through subtracting quality indication through the reference, after which it computing the standard power in the blunder signal.It can be explained as:

$$
\operatorname{MSE}=\frac{1}{M N} \sum_{i=1}^{M} \sum_{j=1}^{N}\left(f(i, j)-f^{\prime}(i, j)\right)^{2}
$$

Where $\mathrm{j}(\mathrm{s})$ is the global normalized histogram of the processed image, $\mathrm{i}$ is the brightness level index, pi is the probability of occurrence of level $i$ in the enhanced image [1].
Table 5.1 Mean Square Error Evaluation

\begin{tabular}{|c|c|c|}
\hline Image name & $\begin{array}{c}\text { Existing } \\
\text { algorithm }\end{array}$ & $\begin{array}{c}\text { Proposed } \\
\text { algorithm }\end{array}$ \\
\hline 1 & 0.0189 & 0.0060 \\
\hline 2 & 0.0621 & 0.0269 \\
\hline 3 & 0.0117 & 0.0173 \\
\hline 4 & 0.0205 & 0.0235 \\
\hline 5 & 0.0428 & 0.0062 \\
\hline 6 & 0.0489 & 0.0150 \\
\hline 7 & 0.0148 & 0.0087 \\
\hline 8 & 0.0650 & 0.0139 \\
\hline 9 & 0.0319 & 0.0044 \\
\hline 10 & 0.0226 & 0.0149 \\
\hline 11 & 0.0142 & 0.0091 \\
\hline 12 & 0.0696 & 0.0204 \\
\hline 13 & 0.0532 & 0.0199 \\
\hline 14 & 0.0486 & 0.0128 \\
\hline 15 & 0.0155 & 0.0082 \\
\hline
\end{tabular}

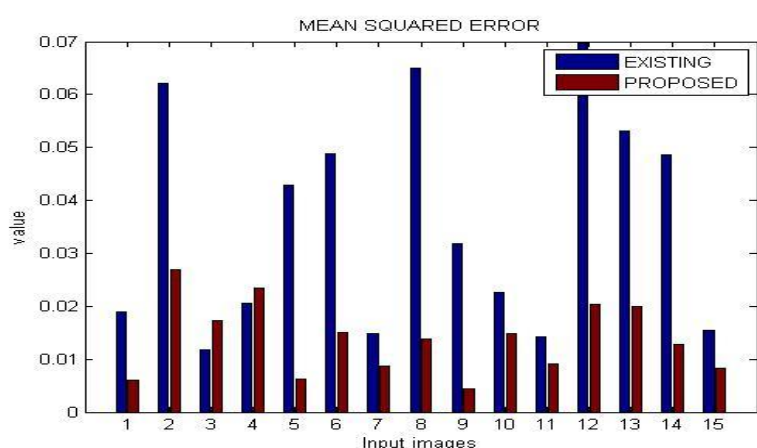

Graph 5.1 MSE of Existing Approach and Proposed Approach for different images

PSNR (peak signal to noise ratio)- Peak square noise ratio is the ratio between the

maximum possible value of the signal and the power of the corrupting noise. It is measured in decibels (db). It can be explained as:

$$
\text { PSNR }=10 . \log _{10}\left(\frac{M A X_{I}^{2}}{M S E}\right)
$$

The average values of all filters are computed in order to calculate percentage improvement. The percentage improvement is expressed as:

$\%$ Improvement $=\frac{\text { Existingaveragevalue }- \text { Proposedaveragevalues }}{\text { Existingvalue }}$

Table 5.2 Peak Signal to Noise Ratio Evaluation

\begin{tabular}{|c|l|l|}
\hline Image name & Existing algorithm & \multicolumn{1}{|c|}{$\begin{array}{c}\text { Proposed } \\
\text { algorithm }\end{array}$} \\
\hline 1 & 65.3600 & 70.3534 \\
\hline 2 & 60.2006 & 63.8330 \\
\hline 3 & 67.4345 & 65.7493 \\
\hline 4 & 65.0186 & 64.4222 \\
\hline 5 & 61.8156 & 70.2278 \\
\hline
\end{tabular}




\begin{tabular}{|l|l|l|}
\hline 6 & 61.2377 & 66.3560 \\
\hline 7 & 66.4235 & 68.7580 \\
\hline 8 & 60.0025 & 66.7072 \\
\hline 9 & 63.0992 & 71.6720 \\
\hline 10 & 64.5867 & 66.3955 \\
\hline 11 & 66.6013 & 68.5564 \\
\hline 12 & 59.7034 & 65.0434 \\
\hline 13 & 60.8755 & 65.1494 \\
\hline 14 & 61.2622 & 67.0503 \\
\hline 15 & 66.2235 & 68.9842 \\
\hline
\end{tabular}

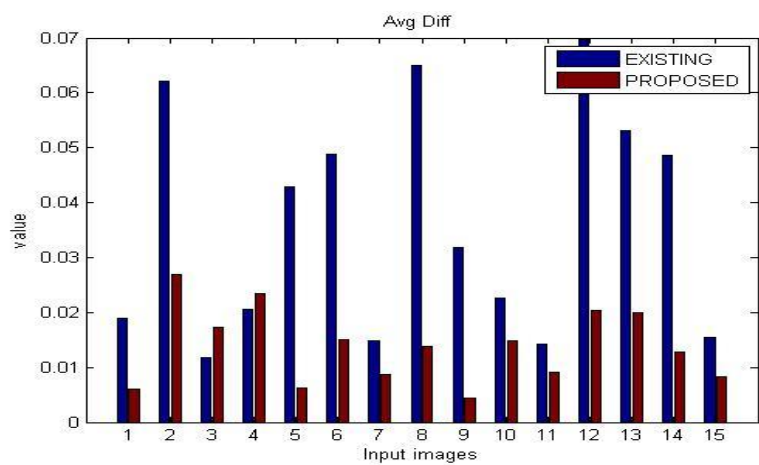

Graph 5.3 Average Difference of Existing Approach \& Proposed Approach for different images

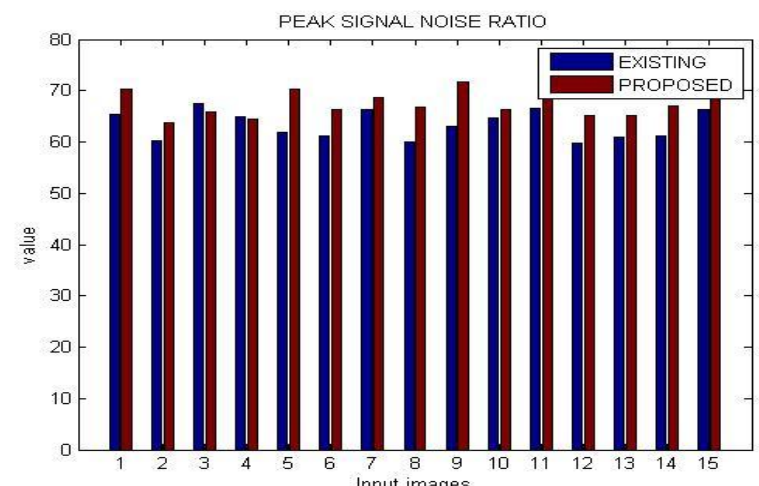

RMSE (Root Mean Square Error) - Root-mean-square error can be a measure on the differences between valuations forecast by means of one or maybe estimator as well as valuations basically observed. It can be explained as:

$$
\text { RMSE }=\sqrt{\frac{1}{M N} \sum_{i=1}^{M} \sum_{j=1}^{N}\left(f(i, j)-f^{\prime}(i, j)\right)^{2}}
$$

Table 5.4.Root Mean Square Error

Table 5.3 Average Difference Evaluation

\begin{tabular}{|l|l|l|}
\hline Image name & \multicolumn{1}{|c|}{$\begin{array}{c}\text { Existing } \\
\text { algorithm }\end{array}$} & \multicolumn{1}{|c|}{$\begin{array}{c}\text { Proposed } \\
\text { algorithm }\end{array}$} \\
\hline 1 & 0.0189 & 0.0060 \\
\hline 2 & 0.0621 & 0.0269 \\
\hline 3 & 0.0117 & 0.0173 \\
\hline 4 & 0.0205 & 0.0235 \\
\hline 5 & 0.0428 & 0.0062 \\
\hline 6 & 0.0489 & 0.0150 \\
\hline 7 & 0.148 & 0.0087 \\
\hline 8 & 0.0650 & 0.0139 \\
\hline 9 & 0.0319 & 0.0044 \\
\hline 10 & 0.0226 & 0.0149 \\
\hline 11 & 0.0142 & 0.0091 \\
\hline 12 & 0.0696 & 0.0204 \\
\hline 13 & 0.0532 & 0.0199 \\
\hline 14 & 0.0486 & 0.0128 \\
\hline 15 & 0.0155 & 0.0082 \\
\hline & & \\
\hline
\end{tabular}

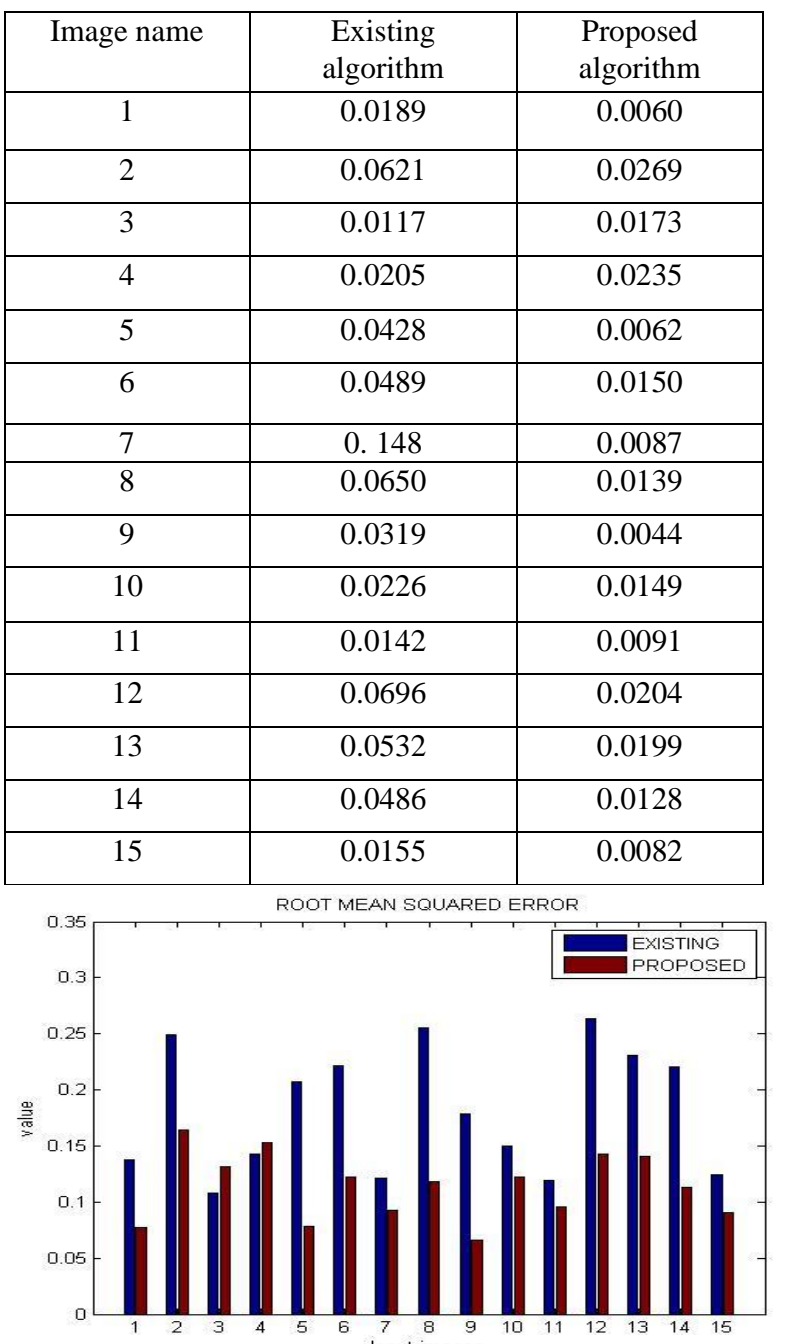

Graph 5.4Root Mean Square Error of Existing Approach \& Proposed Approach for different images 
BER (Bit error rate) -It is defined as the rate in which faults arise inside of a transmission system. This really is immediately converted into the quantity of faults of which arise inside of a chain of a mentioned variety of bits. The definition of bit error rate can be translated into a simple formula:

$$
B E R=\frac{\text { Numberoferrors }}{\text { Totalnumberofbitssent }}
$$

\section{Table 5.5.Bit Error RateRate}

\begin{tabular}{|c|c|c|}
\hline Image name & $\begin{array}{c}\text { Existing } \\
\text { algorithm }\end{array}$ & $\begin{array}{c}\text { Proposed } \\
\text { algorithm }\end{array}$ \\
\hline 1 & 0.0153 & 0.0142 \\
\hline 2 & 0.0166 & 0.0157 \\
\hline 3 & 0.0148 & 0.0152 \\
\hline 4 & 0.0154 & 0.0155 \\
\hline 5 & 0.0162 & 0.0142 \\
\hline 6 & 0.0163 & 0.0151 \\
\hline 7 & 0.0151 & 0.0145 \\
\hline 8 & 0.0167 & 0.0150 \\
\hline 9 & 0.0158 & 0.0140 \\
\hline 10 & 0.0155 & 0.0151 \\
\hline 11 & 0.0150 & 0.0146 \\
\hline 12 & 0.0167 & 0.0154 \\
\hline 13 & 0.0164 & 0.0153 \\
\hline 14 & 0.0163 & 0.0149 \\
\hline 15 & 0.0151 & 0.0145 \\
\hline & & \\
\hline
\end{tabular}

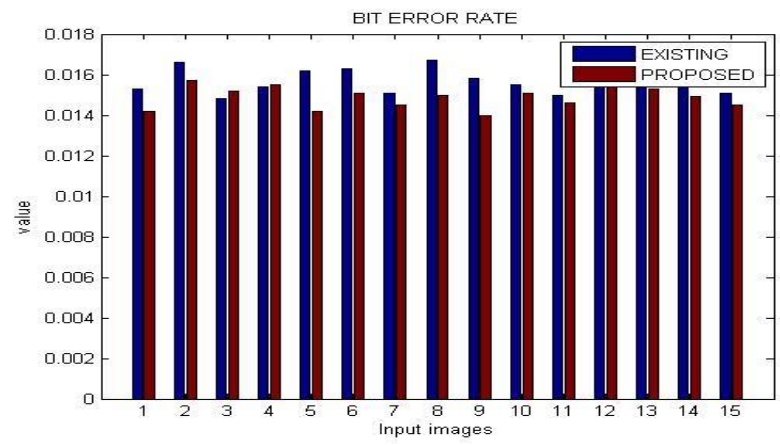

Graph 5.4 Bit error rate of Existing Approach \& Proposed Approach for different images

Cross-Correlation (CC) is a step regarding similarity regarding not one but twosequences as being a function of displacement of a in accordance with other. The idea is generally useful for browsing an extended transmission for any quicker recognized feature.

$$
R x y=\frac{\sum_{i=1}^{n}(x(i)-\bar{x})(y(i)-\bar{y})}{\sqrt{\sum_{i=1}^{n}(x(i)-\bar{x})^{2} \sum_{i=1}^{n}(y(i)-\bar{y})^{2}}}
$$

Table 4. Cross Correlation Evaluation

\begin{tabular}{|c|c|c|}
\hline Image name & $\begin{array}{c}\text { Existing } \\
\text { algorithm }\end{array}$ & $\begin{array}{c}\text { Proposed } \\
\text { algorithm }\end{array}$ \\
\hline 1 & 0.9273 & 0.9409 \\
\hline 2 & 1.5783 & 1.2871 \\
\hline 3 & 1.0334 & 0.9785 \\
\hline 4 & 0.9653 & 0.8995 \\
\hline 5 & 0.9173 & 1.0352 \\
\hline 6 & 0.8248 & 0.9440 \\
\hline 7 & 0.9466 & 0.9531 \\
\hline 8 & 0.8260 & 0.9668 \\
\hline 9 & 1.3570 & 1.0452 \\
\hline 10 & 1.3712 & 1.2400 \\
\hline 11 & 1.1382 & 0.9916 \\
\hline 12 & 0.7680 & 0.9227 \\
\hline 13 & 0.8866 & 0.9475 \\
\hline 14 & 0.8599 & 0.9252 \\
\hline 15 & 0.9946 & 0.9438 \\
\hline
\end{tabular}

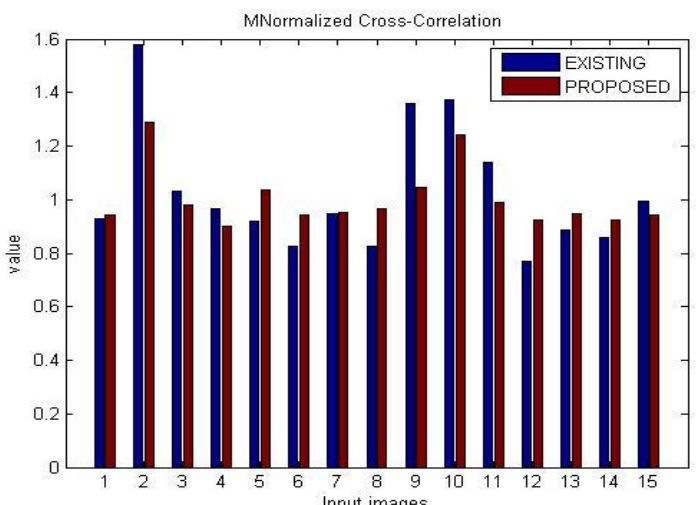

Graph 5.6Cross-Correlation Evaluationof Existing Approach \& Proposed Approach for different images

\section{CONCLUSION AND FUTURE SCOPE}

Image dehazing plays a significant role in image processing applications, to improve the visibility of hazy images. This paper has found that the existing dehazing approaches suffers from over-saturation and edge degradation issue. Also, transform domain method may introduces certain artifacts in restored images. To handle theme issues, in this paper an efficient fuzzy filter based image restoration technique is proposed. Proposed technique is tested on the ten-well-known benchmark hazy images. Comparative analysis of the proposed has clearly demonstrated that it has better structural detail, spatial resolution and rich spectral information than existing dehazing techniques. Mean improvement of the proposed technique is $0.91 \mathrm{~dB}$ and 0.07 for PSNR and SSIM respectively. Thus, the comparison existing technique outperforms over the available enhancement methods. Thus, proposed technique is well suitable for real time dehazing applications. 


\section{REFERENCES}

[1] Wei, Sun, and Han Long,"A New Fast Single-Image Defog Algorithm", Intelligent System Design and Engineering Applications (ISDEA), 2013 Third International Conference on. IEEE, 2013

[2] Tripathi A.K and S. Mukhopadhyay,"Single image fog removal using bilateral filter", Signal Processing, Computing and Control (ISPCC), 2012International Conference on. IEEE, 2012

[3] Nishino, Ko, Louis Kratz, and Stephen Lombardi, "Bayesian defogging", International journal of computer vision , pp 263-278, 2012

[4] Xu Haoran, Guo Jianming, Liu Xing and Ye Lingli, "Fast image dehazing using improved dark channel prior",Information Science and Technology (ICIST), 2012 International Conference on. IEEE, 2012.

[5] Yu, Jing, Chuangbai Xiao, and Dapeng Li, "Physics-based fast single image fog removal", Signal Processing (ICSP), 2010 IEEE 10th International Conference on. IEEE, 2010.

[6] Chu, Chao-Tsung, and Ming-Sui Lee, "A content-adaptive method for single image dehazing", Proceedings of the Advances in multimedia information processing and 11th Pacific Rim conference on Multimedia, Springer-Verlag, 2010.

[7] Guo, Fan, Cai Zixing, Xie Bin and Tang Zin, "Automatic Image Haze Removal Based on Luminance Component", Wireless Communications Networking and Mobile Computing (WiCOM), 2010 6th International Conference on. IEEE, 2010.
[8] Yu, Jing, Chuangbai Xiao and Dapeng Li, "Physics-based fast single image fog removal", Signal Processing (ICSP), 2010 IEEE 10th International Conference on. IEEE, 2010.

[9] Wang, Yan, and Bo Wu, "Improved single image dehazing using dark channel prior", Intelligent Computing and Intelligent Systems (ICIS), 2010 IEEE International Conference on. IEEE, 2010.

[10] Xu, Zhiyuan, and Xiaoming Liu, "Bilinear interpolation dynamic histogram equalization for fog-degraded image enhancement", J Inf Comput Sci 7.8 (2010) 1727-1732.

[11] Wolfe, Christopher, T. C. Graham and Joseph A. Pape, "Seeing through the fog: an algorithm for fast and accurate touch detection in optical tabletop surfaces", ACM International Conference on Interactive Tabletops and Surfaces, 2010.

[12] Chen and Mengyang, "Single image defogging", Network Infrastructure and Digital Content, (NIDC), 2009 International Conference on. IEEE, 2009.

[13] Xu, Zhiyuan, Xiaoming Liu, and Xiaonan Chen,"Fog removal from video sequences using contrast limited adaptivehistogramequalization", Computational Intelligence and Software Engineering, 2009 International Conference on. IEEE, 2009

[14] Desai, Nachiket, Chatterjee Aritra, Mishra Shaunak and Choudary Sunam, "A Fuzzy Logic Based Approach to DeWeather Fog-Degraded Images", Computer Graphics, Imaging and Visualization, 2009 Sixth International Conference on. IEEE, 2009 